\title{
Oseophagus
}

\section{OFR-2 THE SPIT STUDY: CREATING A SALIVA BASED EPIGENETIC BIOMARKER PANEL TO DIAGNOSE OESOPHGEAL CANCER}

\author{
${ }^{1}$ Timothy Stone*, 'Vanessa Ward, ${ }^{2}$ Avi Rosenfeld, 'Aine Hogan, ${ }^{1,3}$ Hazel McBain, \\ 1,3 Margaret Duku, 'Ho K M A, 'Paul Wolfson, 'Ash Wilson, SPIT Study Group The, \\ 1,3 Laurence Lovat. 'University College London, London, UK; ${ }^{2}$ Department of Computer \\ Science, Jerusalem College of Technology, Jerusalem, Israel; ${ }^{3}$ Department of Gastrointestinal \\ Services, University College London Hospitals NHS Foundation Trust, London, UK; ${ }^{4}$ SPIT \\ Study Group
}

\subsection{6/gutjnl-2021-BSG.19}

Introduction The SPIT study aims to investigate the methylation changes in saliva that result from oesophagael cancer with the ultimate aim of developing a diagnostic biomarker.

Methods We recruited volunteers with adenocarcinoma, intramucosal carcinoma, Barrett's Oesophagus and High-Grade Dysplasia. We also recruited two distinct classes of control: healthy volunteers and people with no positive diagnosis of abnormalities after an endoscopy. Study participants completed an extensive questionnaire on relevant lifestyle factors and provided a saliva sample. We had the capacity to analyse 192 saliva samples from our wider cohort using two plates of a standard human methylation array technology. We performed stringent DNA quality-control on our samples and we selected case-control matched samples.

A series of quality-control measures were calculated for the array data. This necessitated the removal of 81 samples, leaving 111 samples for differential methylation analysis. Stringent batch-effect removal was applied by calculating the residuals of a batch-fitted linear model on each locus. Differential methylation analysis was performed on the residuals using standard Bioconductor packages (limma). In order to facilitate generalisation of the results, this process was repeated in a manner similar to hold-out cross validation, where a subcohort generates a model and the remaining left-out samples are used to test it. This was repeated over a thousand times. The loci that were the most often selected were used as a candidate classifier.

Results The 50 probes most frequently selected in repeated sub-sampling showed a marked pattern of separation between controls and adenocarcinoma, but individual probes also showed strong potential to function as a commercially viable biomarker to distinguish between cases and controls. Two probes in particular, which were both strongly implicated in oesophagael cancer in the literature, showed individual diagnostic success accuracies of $87.6 \%$ and $86.2 \%$ (calculated from AUC-ROC values). A candidate biomarker panel using just 4 probes was able to classify held-out samples with an accuracy of $94 \%$, and a panel of 6 probes was able to achieve classification rates of 94\%. Furthermore, this panel of 6 probes appeared to be achieve optimal classification success, as the addition of further probes did not produce any tangible improvement of classification unless a more substantial set of probes were used (24 probes at 95\% accuracy).

The biological plausibility of individual probes was further encouraging. It included probes that were strongly overexpressed in oesophagael cancer, and probes associated with genes implicated in oesophagael cancer survival. An additional probe was, interestingly, associated with a critical tumor- suppressive region with known importance in oesophageal squamous cell carcinoma.

Conclusions We have evidence that a commercially-viable candidate biomarker panel could be implemented to offer a firstpass screening method for oesophagael cancer. This would enable healthcare providers to implement intelligent prioritisation of resources at a time when it is an urgent priority.

\section{OFR-3 EXTENDED WIRELESS PH MONITORING INCREASES GERD DIAGNOSES IN PATIENTS WITH A NORMAL PH IMPEDANCE STUDY}

Sebastian Zeki*, Ismail Miah, Anna Wolak, Minerva deSilva, Jason Dunn, Andrew Davies, Andrew Davies, Abrie Botha, Guiping Sui, Jafar Jafari, Terry Wong. Guy's And St Thomas' NHS Trust, London, UK

\subsection{6/gutjnl-2021-BSG.20}

Introduction Prolonged acid reflux monitoring using wireless $\mathrm{pH}$ monitoring (WPM) may be performed following $\mathrm{pH}$ impedance in suspected false negative cases. The increased yield of Gastro-esophageal reflux disease (GERD)-positive diagnoses with WPM is unknown. Also unknown is which parameters from the negative $\mathrm{pH}$ impedance or manometry, may predict a subsequent positive WPM test. Aim: a) Determine the increased yield and b) determine predictors of GERD positivity in patients with GERD-negative $\mathrm{pH}$ impedance.

Methods The increased GERD-positive diagnostic yield with WPM was determined in 181 consecutive patients who had undergone negative $\mathrm{pH}$ impedance with HRM. Univariate and multivariate analysis determined predictors of a positive WPM. Results Of the 181 included, using average day analysis 61 studies were positive for GERD (Male : Female 26: 35, average age of 47 (15) years and 120 negative for GERD (Male: Female 52: 68 average age of 48 (15) years. This corresponds to $33.70 \%$ of the patients initially diagnosed as not having GERD, being given a diagnosis of GERD at WPM.

Using a worst day analysis, of the patients who were negative for GERD at impedance, 62 studies were positive at WPM (34.25\%). 92\% of the patients diagnosed with GERD on worst day analysis were also positive on average day analysis. $20(33.33 \%)$ of patients classified as WPM-GERD-positive were positive for only 1 day. The number of studies that were negative for GERD in the first 24 hours and 48 hours of the WPM study was $19(31.15 \%)$ and $11(18.03 \%)$ respectively.

Using univariate analysis, significant differences were found for basal respiratory minimum $(\mathrm{mmHg})$ (GERD positive: 8 (8),GERD negative: 10 (8) $\mathrm{p}=0.010)$, number of acid episodes (GERD positive:20 (14) GERD negative: 12 (11) $\mathrm{p}=0.001$ ) and acid exposure time (GERD positive:1.70 (1.35) GERD negative: $1.07(1.14) \mathrm{p}=0.002)$ on univariate analysis between the groups. Only the basal respiratory minimum was significantly associated with a WPM-GERD positive result on multivariate analysis (OR:0.95 (0.90, 1.00)).

Conclusions There is a significant increased yield for GERD positive diagnoses with WPM after a negative impedance study which may be related to the presence of intermittent reflux in these patients. Predictability is difficult to ascertain from HRM or 24-hour $\mathrm{pH}$ impedance variables. The increased yield suggests a longer period of assessment for acid reflux rather than for different refluxate components may be 